\title{
Surface Instabilities in Cracks
}

\author{
Efim A. Brener ${ }^{1}$ and V. I. Marchenko ${ }^{2}$ \\ ${ }^{1}$ Institut für Festkörperforschung, Forschungszentrum Jülich, D-52425 Jülich, Germany \\ ${ }^{2}$ P. L. Kapitza Institute for Physical Problems, RAS, 117334, Kosygin street 2, Moscow, Russia
}

(Received 13 August 1998)

The surface of a propagating crack is shown to be morphologically unstable because of the nonhydrostatic stresses near the surface (Asaro-Tiller-Grinfeld instability). We find that the energy of a wavy crack becomes smaller than the energy of a straight crack if the crack length is a few times larger than the Griffith length. The local dispersion relation is derived assuming that the instability develops via mass transport by surface diffusion. We also argue that the widely used condition of the vanishing of $K_{\mathrm{II}}$, the stress-intensity factor of the sliding mode, appears in a natural way in our description as an effective boundary condition at the tip of the crack. [S0031-9007(98)07834-X]

PACS numbers: 46.30.Nz, 62.20.Mk, 81.40.Np

The uniform motion of a straight crack is well understood [1]. Experiments on the fracture of bulk specimens, however, show that the crack surfaces are often rough [2]. Some of these results are interpreted in the framework of models of cracks propagating in heterogeneous media. The other possibility for the roughening of the crack surfaces is the instability of the straight motion of the crack tip. Recent experiments on the fracture of thin plates [3] clearly established that many puzzling phenomena in brittle fracture dynamics are related to an oscillatory instability at velocities appreciably below the Rayleigh speed $V_{R}$. Beyond a critical velocity the crack dynamics change dramatically. At that point, the mean acceleration of the crack drops, the crack velocity starts to oscillate, and a pattern correlated with the velocity oscillation is created on the fracture surface. These results stimulated many recent investigations (see, for example, Ref. [4]) but the instability is not well understood yet.

There were several attempts in literature to investigate the stability of the propagating cracks. The linear stability analysis of the quasistatic crack subject to mode I (opening-mode) loading has been performed by Cotterell and Rice [5] with subsequent refinement by Adda-Bedia and Ben Amar [6]. They employ the Griffith theory and the so-called principle of local symmetry, i.e., the condition that the mode II (sliding-mode) stress-intensity factor $K_{\text {II }}$ vanishes on the tip of the crack. They found that the straight motion of the crack becomes unstable if the tangential loading exceeds a critical value.

A fully dynamical model, including the microscopic description of the cohesive zone of the crack tip, has been discussed by Ching, Langer, and Nakanishi [7]. The cohesive force in the neighborhood of the tip provides a fracture energy and a mechanism for regularizing the stress singularity. The use of such a model removes the need to speculate about a principle of local symmetry. In addition to the results of Refs. [5,6], they found a strong microscopic instability even for very low crack velocities. The main reason for such a strong instability is that the tangential stress, which deflects the crack away from a straight motion, exceeds the normal stress on the fracture surface throughout the tip region at all nonzero velocities. This instability is very sensitive to tiny details of the cohesive-zone model.

In all of these descriptions, a crack surface is viewed as the trace left behind by the crack tip as it traverses the sample. All modes related to the further surface deformations due to a transfer of matter are assumed to be frozen. The main purpose of this Letter is to describe the instabilities of the crack surface related to these so far missing degrees of freedom. We will find that the surfaces of a propagating crack undergo an Asaro-TillerGrinfeld (ATG) instability [8,9] of purely macroscopic origin.

Asaro and Tiller [8] and later Grinfeld [9] brought out the idea that the surface of a solid which is subject to a uniaxial stress (i.e., when $\sigma_{0}=\sigma_{x x}-\sigma_{y y} \neq 0$ ) is morphologically unstable. This instability has an elastic origin: a corrugated surface leads to a lower elastic energy than a flat one. Note that the instability implies a transfer of matter, either from a liquid phase or through surface diffusion. The interface displacement is due to redistribution of material, not due to elastic strain. Surface energy prevents short-scale deformations. The chemical potential of such a solid near the surface can be written as $[10,11]$

$$
\mu_{s}=v_{s}\left[f_{s 0}+\frac{1-\nu^{2}}{2 E}\left(\sigma_{\tau \tau}-\sigma_{n n}\right)^{2}+\alpha K\right] .
$$

Here, $v_{s}$ is the atomic volume of the solid phase; $f_{s 0}$ is the free energy density for a hydrostatic situation; $\alpha$ is the surface energy; $K$ is the curvature of the interface (counted positive for a convex solid); $\sigma_{i k}$ is the stress tensor; $n$ and $\tau$ are subscripts referring to the normal and the tangent to the interface; $\nu$ and $E$ are the Poisson and Young coefficients. For a small perturbation of the interface $\left(y(x, t)=Y_{k} \exp [i k x+\omega t]\right)$, the shape 
dependent part of the chemical potential is [9]

$$
\mu_{s}=v_{s}\left[\frac{2\left(1-\nu^{2}\right) \sigma_{0}^{2}}{E}|k|-\alpha k^{2}\right] y(x, t) .
$$

If the solid is in contact with a liquid which provides a reservoir of atoms, the instability manifests itself as a melting-crystallization process; the normal velocity of the interface is proportional to the difference of chemical potentials between the solid and liquid. If the surface is the interface between the solid and vacuum, the instability develops via surface diffusion; the normal velocity is proportional to the Laplacian of the chemical potential of the solid phase. In the latter case, this leads to the following dispersion relation:

$$
\omega=D k^{2}\left[\frac{2 \sigma_{0}^{2}\left(1-\nu^{2}\right)}{\alpha E}|k|-k^{2}\right],
$$

where $D$ is proportional to the surface diffusivity. Here, elasticity plays a destabilizing role and $|k|$ reflects its nonlocal properties.

Let us discuss a two-dimensional crack subject to mode I (opening-mode) loading at infinity perpendicular to the crack: $\sigma_{y y}^{\infty}=P$. This crack is stable in the sense of Refs. $[5,6]$. However, the surface of the crack behind the moving tip becomes unstable. The crucial observation is that at the crack surface $\sigma_{n n}=0$ but $\sigma_{\tau \tau}$ is not. Thus, we can expect the development of the ATG instability (we note that hydrostatic loading is not favorable for the ATG instability). However, the application of this theory to the surface of the crack requires some comments and modifications. We are not in a homogeneous situation due to the presence of the finite crack. The stress field is singular and should be found from the solution of the corresponding crack problem.

In a quasistatic case, which we discuss first, the stress field in the close vicinity of the crack surface is given by (see, for example, Ref. [12])

$$
\begin{gathered}
\sigma_{x x}=-P\left[1-2 L^{2} y /\left(L^{2}-x^{2}\right)^{3 / 2}\right], \\
\sigma_{y y}=0 \text { and } \sigma_{x y}=0 .
\end{gathered}
$$

Here, we assume that the straight crack of the length $2 L$ is located at $-L<x<L$ and $y /(L-x) \ll 1$.

Because of the presence of the singular stress field, there is an additional elastic contribution to the chemical potential [Eq. (2)] on the surface of the corrugated crack $y(x)$ :

$$
\delta \mu_{s}=-\frac{2 v_{s} P^{2}\left(1-\nu^{2}\right) L^{2}}{E\left(L^{2}-x^{2}\right)^{3 / 2}} y .
$$

This stabilizing $k$-independent term plays the role of effective "gravity" (in terms of the customary ATG instability) but it depends upon the distance from the tip. Combining together Eqs. (2) and (5), we obtain the modified ATG spectrum:

$$
\omega=D k^{2}\left[\frac{2 P^{2}\left(1-\nu^{2}\right)}{\alpha E}\left(|k|-\frac{L^{2}}{\left(L^{2}-x^{2}\right)^{3 / 2}}\right)-k^{2}\right] .
$$

This local dispersion relation is valid for relatively shortscale deformations, $k r \gg 1$, where $r=(L-x)$ is the distance from the tip.

The instability occurs at distances $r>r_{0} \sim L_{G} \times$ $\left(L / L_{G}\right)^{1 / 3}$ and at $k_{c} \sim 1 / L_{G}$. Here, we have introduced the so-called Griffith length:

$$
L_{G}=\frac{2 E \alpha}{\pi\left(1-\nu^{2}\right) P^{2}} .
$$

The local dispersion relation (6) quantitatively describes the instability if $L \gg L_{G}$.

Noziéres [11] concluded from a weakly nonlinear analysis of the customary ATG instability that the bifurcation from the flat surface into the corrugated one is subcritical: the first nonlinearity reinforces the instability. This result points to the fact that amplitude expansions are inadequate and, therefore, one has to perform a fully nonlinear analysis in order to describe the subsequent development of the instability. The numerical nonlinear analysis of the customary ATG instability $[13,14]$ shows that even close to the instability threshold deep cracklike grooves form. These grooves, pointing into the solid, sharpen and accelerate as they deepen. Therefore, a complicated pattern should develop behind the moving tip.

In order to find the critical length of the straight crack at which the crack undergoes the ATG instability we must go beyond the local approximation which has been used so far. We have to calculate the change of the energy up to the second order with respect to the perturbation of the center line $y(x)$ and to find the critical length when this energy change becomes negative for the first time. The most trivial contribution is due to the surface energy

$$
U_{s}=2 \alpha \int_{-L}^{L} \frac{\left(y^{\prime}\right)^{2}}{2} d x,
$$

which is, of course, always positive and stabilizes the straight crack. The other stabilizing contribution is of elastic origin and can be read off directly from Eq. (5):

$$
\begin{aligned}
U_{\mathrm{e} 1 s} & =-2 \int_{-L}^{L} \frac{\delta \mu_{s}}{v_{s}} \frac{y(x)}{2} d x \\
& =\int_{-L}^{L} d x \frac{4 P^{2}\left(1-\nu^{2}\right) L^{2}}{E\left(L^{2}-x^{2}\right)^{3 / 2}} \frac{y^{2}}{2} .
\end{aligned}
$$

The factors 2 in Eqs. (8) and (9) come from the fact that the crack has two surfaces. Assuming that $y=0$ at $x= \pm L$ and integrating by part, we can remove the singularities in Eq. (9):

$$
U_{\mathrm{el} s}=-\frac{2 P^{2}\left(1-\nu^{2}\right)}{E} \int_{-L}^{L} d x\left(y^{2}\right)^{\prime \prime} \sqrt{L^{2}-x^{2}} .
$$

The destabilizing contribution to the elastic energy which corresponds to the ATG instability in our finite length 
crack geometry can be calculated as follows. The total shear stress on the crack surface must be zero:

$$
\sigma_{\tau n}=\sigma_{\tau n}^{(1)}+P y^{\prime}=0,
$$

where $\sigma_{i k}^{(1)}$ is the first order correction to the stress field which must be added to satisfy this boundary condition. Associated with this field the mode II (sliding-mode) stress-intensity factor $K_{\text {II }}$ was calculated in Refs. [5,6]:

$$
K_{\mathrm{II}}=\frac{P}{\sqrt{\pi L}} \int_{-L}^{L} d x \sqrt{\frac{L+x}{L-x}} y^{\prime} .
$$

The corresponding elastic energy $U_{\mathrm{el} u}$ can be found by using the well-known relation established by Irwin [15]:

$$
-\frac{\partial U_{\mathrm{e} l u}}{\partial L}=\frac{2\left(1-\nu^{2}\right)}{E} K_{\mathrm{II}}^{2} .
$$

We note that another contribution to $K_{\mathrm{II}}$, which is just $y^{\prime}(L) K_{\mathrm{I}} / 2$, has been already taken into account by Eqs. (5) and (9) (here, $K_{\mathrm{I}}$ is mode I stress-intensity factor for the straight crack, $K_{\mathrm{I}}=P \sqrt{\pi L}$ ).

The total energy from Eqs. (8), (10), and (13),

$$
U=U_{s}+U_{\mathrm{el} s}+U_{\mathrm{el} u},
$$

is a complicated functional of the perturbation $y(x)$ and depends also on the length of the crack. This expression for the energy of the corrugated crack is consistent with the result of Ref. [16].

The optimal perturbation corresponds to the minimum of this functional and the threshold of the instability corresponds to the condition $U_{\min }=0$. We note that the optimal perturbation corresponds to the condition of constant chemical potential $\mu_{s}$ along the crack surfaces.

In order to estimate the critical length, we use the variational procedure with a simple representation of the perturbation,

$$
y(x)=Y_{k}(\cos k x-\cos k L) .
$$

Then all integrals can be performed analytically, and we find for Eq. (14)

$$
U=\frac{\pi P^{2}\left(1-\nu^{2}\right)}{E}(k L)^{2} F(k L) Y_{k}^{2},
$$

where the function $F(z)$ can be expressed in terms of Bessel functions $J_{m}$ :

$$
\begin{aligned}
F(z)= & \left(L_{G} / 2 L\right)[1-\sin 2 z / 2 z]-\left[J_{0}^{2}(z)+J_{1}^{2}(z)\right] \\
& +2\left[J_{1}(2 z)-2 J_{1}(z) \cos z+J_{0}(z) J_{1}(z)\right] / z .
\end{aligned}
$$

For $k L \gg 1$, this energy is consistent with the spectrum (3) of the customary ATG instability. For $k L \ll 1$, the result is in agreement with an exact solution of the circular arc crack (see, for example, Ref. [5]). A straight crack is stable if $U>0$. This is always the case for small $L$. The bifurcation threshold corresponds to $U=0$ and $d U / d k=0$ (see Fig. 1). Thus, the straight crack becomes unstable at $L_{c} \approx 6 L_{G}$ and $k_{c} L_{G} \approx 0.7$, where

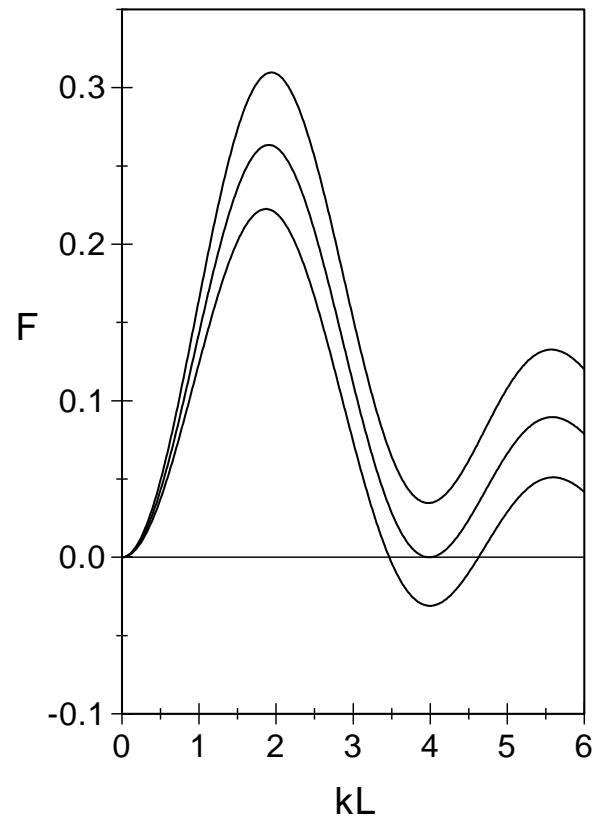

FIG. 1. The reduced energy $F$ versus $k L$ for three different values of the length of the crack; from the bottom to the top $L=10 L_{G}, L=5.85 L_{G}, L=4 L_{G}$. The crack goes unstable near $k L=4$ where $F<0$.

$L_{G}$ is the Griffith length [Eq. (7)]. The perturbations of the form $\sin (n \pi x / L)$ give the bifurcation threshold $L_{c} \approx 11 L_{G}$ for $n=2$.

In calculating the energy of the corrugated crack, we have discussed only the perturbation of the center line. This assumes that both surfaces of the crack are perturbed in the same way such that the crack can be still described as an ideal mathematical cut. However, the development of the instability in the nonlinear regime leads to the formation of the deep grooves pointed into the solid $[13,14]$. Thus, the corrugation of the opposite surfaces of the crack becomes incoherent.

In the quasistatic approximation used so far we have assumed a slow motion of the crack tip. In the framework of a thermodynamical description we find pure energetic reasons for the instability of the crack surfaces. Including surface diffusion as a mechanism for the mass transport, we find the local dispersion relation (6) which describes the initial stage of the development of the instability.

Let us discuss dynamical aspects of the instability when the tip velocity is not assumed to be small. It is known from fracture dynamics [1] that the stress distribution on the crack surface remains qualitatively the same as in the static case apart from some factors which depend on the tip velocity $V_{t}$. Therefore, instead of the quasistatic spectrum (6) we can write the spectrum in the laboratory frame of reference as

$$
\omega=D k^{2}\left[\frac{4}{\pi L_{G}}\left(q_{1}|k|-\frac{q_{2} L^{2}}{\left(L^{2}-x^{2}\right)^{3 / 2}}\right)-k^{2}\right],
$$


where $q_{1}$ and $q_{2}$ are some functions of $V_{t} / V_{R}$ and the Poisson ratio $\nu$.

We can expect that the linear instability described by the local dispersion relation (17) should be only convective in the frame of reference of the moving tip due to its slow development compared to the fast tip motion. Indeed, the most unstable mode corresponds to the value of $k \sim$ $q_{1} / L_{G}$ and $\omega=\omega_{u} \sim D q_{1}^{4} / L_{G}^{4}$. In the moving frame of reference, $\omega$ should be replaced by $\left(\omega-i V_{t} k\right)$ with a convective contribution of the order $\omega_{c} \sim q_{1} V_{t} / L_{G}$. The ratio $\omega_{u} / \omega_{c} \sim q_{1}^{3} D /\left(L_{G}^{3} V_{t}\right)$ is expected to be small if the velocity $V_{t}$ is of the order of the sound speed. This corresponds only to the convective instability (see, for example, Ref. [17]). According to the concept of the convective instability, it means that tip motion is insensitive to the development of the instability behind the tip. However, the drastic acceleration of the instability and the refining of the length scale in the nonlinear regime $[13,14]$ make still conceivable that eventually the tip motion itself could be affected by the instability.

The pattern which develops on the surface of the main crack in the recent experiments on the microbranching instability [3] superficially looks similar to the pattern predicted by the nonlinear ATG instability. However, the direct relevance of our results to these experiments is problematic because of the slow surface diffusion process compared to the fast tip motion.

The described instability has a purely macroscopic origin (compared to the microscopic tip instability predicted in Ref. [7]) and it does not depend on details of the cohesive or plastic zone. As it follows from our analysis the instability should be more efficient in the case of slow cracks.

Finally, we note that the widely used condition of the vanishing of the total $K_{\text {II }}$, the sliding-mode, stressintensity factor, appears in a natural way in our description as an effective boundary condition at the tip of the crack. Indeed, if the total $K_{\mathrm{II}}$ is not zero, there would be divergent contributions to the chemical potential on the crack surfaces in the vicinity of the tips. These contributions have the opposite signs on the opposite surfaces. On the other hand, these pieces of the crack surface are separated only by a microscopic distance and the mass transport should be very fast on this scale. This leads to the natural boundary condition that the chemical potentials on the opposite surfaces of the crack in the vicinity of the tip should be equal. In turn, it requires that $K_{\text {II }}^{\text {tot }}=0$. We stress that even if the surface diffusion process is slow on the macroscopic scale of the length of the crack it can be very efficient on the microscopic scale in the vicinity of the crack tip.
We appreciate support by a European Commission travel grant "Procope." Stimulating discussions with S. Iordanskii, H. Müller-Krumbhaar, J.R. Rice, and Y. Saito are greatly appreciated. V.I.M. thanks Forschungszentrum Jülich for its hospitality.

[1] L. B. Freund, Dynamic Fracture Mechanics (Cambridge University Press, New York, 1990).

[2] E. Bouchaud, J. Phys. Condens. Matter 9, 4319 (1997); P. Daguier, B. Nghiem, E. Bouchaud, and F. Greuzet, Phys. Rev. Lett. 78, 1062 (1997); S. Ramanathan, D. Ertas, and D.S. Fisher, Phys. Rev. Lett. 79, 873 (1997); S. Ramanathan and D. S. Fisher, Phys. Rev. Lett. 79, 877 (1997), and references therein.

[3] J. Fineberg, S. P. Gross, M. Marder, and H. L. Swinney, Phys. Rev. Lett. 67, 457 (1991); Phys. Rev. B 45, 5146 (1992); S. P. Gross, J. Fineberg, M. Marder, W. D. McCormick, and H. Swinney, Phys. Rev. Lett. 71, 3162 (1993).

[4] F. Abraham, D. Brodbeck, R. A. Rafey, and W. E. Rudge, Phys. Rev. Lett. 73, 272 (1994); J. R. Rice, Y. Ben-Zion, and K. Kim, J. Mech. Phys. Solids 42, 813 (1994); M. Marder and S. Gross, J. Mech. Phys. Solids 43, 1 (1995); J. F. Boudet, S. Ciliberto, and V. Steinberg, Europhys. Lett. 30, 337 (1995); J. Phys. II (France) 6, 1493 (1996); E. Sharon, S. P. Gross, and J. Fineberg, Phys. Rev. Lett. 74, 5096 (1995); Phys. Rev. Lett. 76, 2117 (1996); E. Sharon and J. Fineberg, Phys. Rev. B 54, 7128 (1996); F. Lund, Phys. Rev. Lett. 76, 2742 (1996); J. F. Boudet and S. Ciliberto, Phys. Rev. Lett. 80, 341 (1998).

[5] B. Cotterell and J. R. Rice, Int. J. Fract. 16, 155 (1980).

[6] M. Adda-Bedia and M. Ben Amar, Phys. Rev. Lett. 76, 1497 (1996).

[7] E. S. C. Ching, J. S. Langer, and H. Nakanishi, Phys. Rev. E 53, 2864 (1996).

[8] R. J. Asaro and W. A. Tiller, Metall. Trans. 3, 1789 (1972).

[9] M. A. Grinfeld, Dokl. Akad. Nauk. SSSR 290, 1358 (1986) [Sov. Phys. Dokl. 31, 831 (1986)].

[10] C. Herring, J. Appl. Phys. 21, 437 (1950).

[11] P. Noziéres, J. Phys. I (France) 3, 681 (1993).

[12] J. R. Rice, in Fracture: An Advanced Treatise, edited by H. Liebowitz (Academic, New York, 1968), Vol. 2, Chap. 3, pp. 191-311.

[13] W. H. Yang and D. J. Srolovitz, Phys. Rev. Lett. 71, 1593 (1993).

[14] K. Kassner and C. Misbah, Europhys. Lett. 28, 245 (1994).

[15] G. R. Irwin, J. Appl. Mech. 24, 361 (1957).

[16] M. Adda-Bedia and Y. Pomeau, Phys. Rev. E 52, 4105 (1995).

[17] E.M. Lifshitz and L.P. Pitaevskii, Physical Kinetics (Pergamon Press, Oxford, 1981). 